\title{
Safety and Efficacy of Once-Daily Administration of Mycophenolate Mofetil in Kidney Transplant Patients-A New Treatment Option for Non-Adherence*
}

\author{
Naotsugu Ichimaru ${ }^{1 \#, ~ K a z u a k i ~ Y a m a n a k a ², ~ T a i g o ~ K a t o ², ~ Y o i c h i ~ K a k u t a ², ~ T o y o f u m i ~ A b e ², ~}$ \\ Jun-ya Kaimori', Ryoichi Imamura², Norio Nonomura ${ }^{2}$, Shiro Takahara1 \\ ${ }^{1}$ Department of Advanced Technology for Transplantation, Osaka University Graduate School of Medicine, \\ Suita, Japan \\ ${ }^{2}$ Department of Specific Organ Regulation (Urology), Osaka University Graduate School of Medicine, Suita, Japan \\ Email: \#ichimaru@att.med.osaka-u.ac.jp
}

Received 5 June 2014; revised 20 July 2014; accepted 1 August 2014

Copyright $@ 2014$ by authors and Scientific Research Publishing Inc. This work is licensed under the Creative Commons Attribution International License (CC BY). http://creativecommons.org/licenses/by/4.0/ (c) () Open Access

\section{Abstract}

Non-adherence to immunosuppressive therapy is associated with reduced graft survival. Fifteen long-term follow up kidney transplant patients with stable post-transplant clinical courses were enrolled in the study. All patients were prescribed to tacrolimus prolonged release, mycophenolate mofetil (MMF) and corticosteroid at the time of enrolment. Twice-daily administration of MMF was then converted to a single daily dose. As a result, all immunosuppressive agents were administered simultaneously at one time in the morning. The daily total doses of the three immunosuppressants were identical during the study period. No acute rejection or adverse event was observed during the study period. Blood urea nitrogen and estimated glomerular filtration rate did not change significantly after conversion to once-daily administration. The blood tacrolimus trough level and the Mycophenolic acid (MPA) trough level also did not change significantly after conversion to once-daily administration. Interestingly, the meanmedian MPA trough level remained $>3 \mu \mathrm{g} / \mathrm{ml}$ even after conversion to once-daily administration. A sufficient plasma MPA level was maintained after conversion to once-daily administration, and no acute rejection was observed during the study period. To our knowledge, this study is the first to report that the plasma MPA concentration can be maintained after once-daily administration of MMF in long-term kidney transplant patients. Once-daily administration of immunosuppressive agents may improve longterm graft survival because of better treatment adherence due to the reduced dosing frequency.

\footnotetext{
"Grants received: NI, JK, ST (Astellas Pharma, Novartis Pharma and Chugai Pharmaceutical).

"Corresponding author.
} 
The safety or efficacy of conversion to once-daily administration of MMF should be evaluated in a future randomized controlled large-scale clinical study.

\title{
Keywords
}

\author{
Once-Daily Administration, Mycophenolate Mofetil, Treatment Adherence
}

\section{Introduction}

Non-adherence to immunosuppressive therapy is associated with reduced 5-year graft survival [1]. The odds of graft failure are sevenfold greater in non-adherent patients versus adherent patients, and non-adherence is considered as the leading avoidable cause of graft failure [2]. In this prospective clinical study, we evaluated the safety and efficacy of conversion from twice-daily to once-daily administration of mycophenolate mofetil (MMF) in stable long-term kidney transplant patients.

\section{Patients and Methods}

Fifteen long-term follow up kidney transplant patients at Osaka University Hospital (Osaka, Japan) and Inoue Hospital (Suita, Japan) with stable post-transplant clinical courses were enrolled in the study. All patients were prescribed tacrolimus prolonged release (TAC-PR), MMF and corticosteroid (CS) at the time of enrolment. Twice-daily administration of MMF was then converted to a single daily dose. As a result, all immunosuppressive agents were administered simultaneously at one time in the morning. The daily total doses of the three immunosuppressants were identical during the study period. Acute rejection rate, kidney graft function, blood level of tacrolimus (TAC) and MMF, as well as other adverse events were monitored from 3 months prior to 3 months after conversion. We used repeated-measures analysis of variance followed by Dunnett's $t$-test to compare the laboratory data, and a $p$ value of $<0.05$ was considered significant using STATA/SE 13.0 for Windows (STATA Corp. LP, College Station, TX). The Institutional Review Board at Osaka University Hospital (no. 10287) and Inoue Hospital (no. 173) approved the study protocol. The study was registered in the University Hospital Medical Information Network Clinical Trials Registry (UMIN000008985).

\section{Results}

Of the 15 patients enrolled in the study, 10 were male and 5 were female with a mean age of 48 years. On average, 8.4 years had passed since kidney transplant surgery. Table 1 summarizes the mean drug doses administered to enrolled patients; the mean TAC-PR dose was $2.2 \mathrm{mg} /$ day, the mean prednisolone dose was $4.3 \mathrm{mg} / \mathrm{day}$, and the mean MMF dose was $950 \mathrm{mg} /$ day with most patients taking $1000 \mathrm{mg}$ MMF/day (Table 1). As a result, no acute rejection or adverse event was observed during the study period. Blood urea nitrogen and estimated

\begin{tabular}{ccc} 
Table 1. Patients' characteristics. & \\
\hline Gender (male/female) & $10 / 5$ & \\
Age (years) & $48 \pm 11.1$ & $(34-72)$ \\
Body weight (kg) & $59.2 \pm 11.4$ & $(37.0-77.0)$ \\
Height (cm) & $167.5 \pm 7.5$ & $(150-180)$ \\
BMI (kg/m²) & $20.9 \pm 3.0$ & $(16.4-27.6)$ \\
Years from Tx & $8.4 \pm 3.6$ & $(3.4-17.9)$ \\
TAC-PR (mg/day) & $2.2 \pm 0.9$ & $(1.0-4.0)$ \\
MMF (mg/day) & $950 \pm 101$ & $(750-1000)$ \\
Prednisolone (mg/day) & $4.3 \pm 1.2$ & $(1.25-5.0)$ \\
\hline
\end{tabular}

Data are presented as means \pm standard deviation (minimum-maximum values). BMI, body mass index; MMF, mycophenolate mofetil; TAC-PR, tacrolimus prolonged release; Tx, transplantation. 
glomerular filtration rate did not change significantly after conversion to once-daily administration. The blood TAC trough level and the Mycophenolic acid (MPA) trough level also did not change significantly after conversion to once-daily administration. Interestingly, the median MPA trough level remained $>3 \mu \mathrm{g} / \mathrm{ml}$ even after conversion to once-daily administration (Figure 1).

\section{Discussion}

We have reported previously that the adherence rate decreased with time following kidney transplantation for both the morning and evening doses, and the decrease in adherence rate was more pronounced with the evening doses. Moreover, a majority of patients (89.1\%) responded that they would prefer a decreased dosing frequency at the time of the study [3]. In 2013, we also reported that a single daily dose might improve treatment adherence and kidney allograft survival [4]. In this study, we prescribed TAC-PR, MMF and CS once-daily in the morning after conversion from a twice-daily regimen. With this dosing regimen, kidney graft function remained stable and no acute rejection or other adverse events occurred within 3 months after conversion to once-daily administration. However, the long-term results of once-daily administration should be assessed in future studies. The plasma MPA concentration of oral MMF shows a sharp peak at 1 hour and a secondary peak at $8-12 \mathrm{~h}$. The elimination half-life of MPA after administration of an oral single-dose of 1.5-g MMF to 12 healthy volunteers was $17.9 \mathrm{~h}$ with a clearance of $11.6 \mathrm{~L} / \mathrm{h}$ [5]. In our study, the median plasma MPA trough level did not change significantly after conversion to once-daily administration. To evaluate the pharmacokinetics of oncedaily administration of MMF in depth, a 24-h pharmacokinetics of MPA would need to be conducted. The fact that the median MPA plasma trough level was maintained at $>3 \mu \mathrm{g} / \mathrm{ml} 3$ months after conversion to once-daily administration was worthy of special mention. A sufficient plasma MPA level was maintained after conversion to once-daily administration, and no acute rejection was observed during the study period. To our knowledge, this study is the first to report that the plasma MPA concentration can be maintained after once-daily administration of MMF in long-term kidney transplant patients. Our study is limited as a single-arm, single-center small
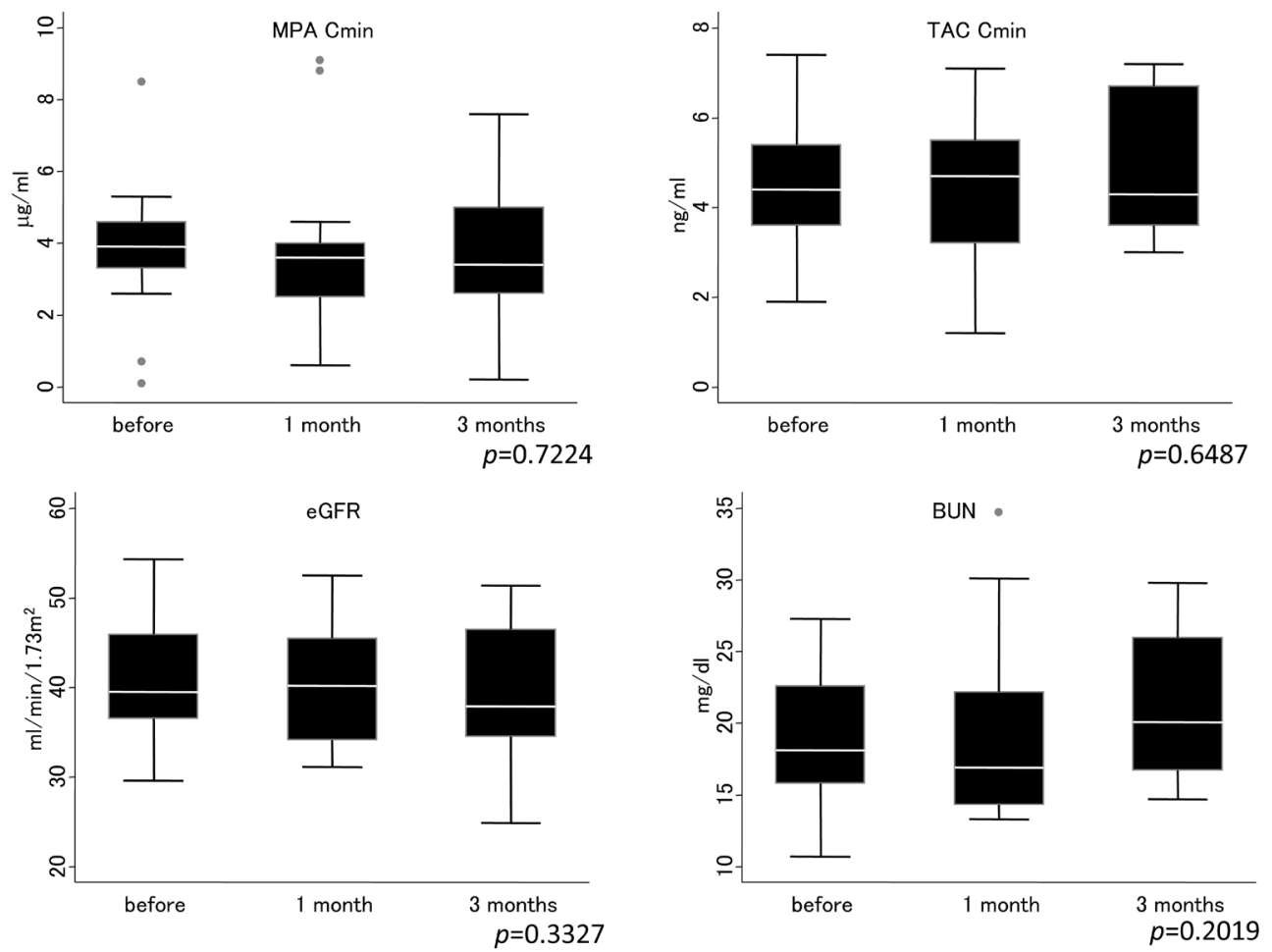

Figure 1. Box plots of laboratory data before, one month after, and three months after conversion. Blood urea nitrogen (BUN) and estimated glomerular filtration rate (eGFR) did not change significantly after conversion to once-daily administration. The blood tacrolimus (TAC) trough level (Cmin) and the Mycophenolic acid (MPA) trough level also did not change significantly after conversion to once-daily administration. 
case study with a relatively short observation period. Long-term follow-up is needed, particularly for evaluation of treatment adherence. Concomitant non-immunosuppressive agents prescribed for hypertension, hyperlipidemia, or diabetes were not evaluated. Only patients with long-term stable clinical course were enrolled in the study, and patients with recent rejection episodes or progressively deteriorating kidney graft function were not included. The safety or efficacy of conversion to once-daily administration of MMF for such high-risk kidney transplant patients remains unknown and should be evaluated in a future randomized controlled large-scale clinical study.

\section{Conclusion}

Conversion to once-daily administration of TAC-PR, MMF and CS in long-term kidney transplant patients with stable clinical course provided a safe short-term outcome. Once-daily administration of immunosuppressive agents may improve long-term graft survival because of better treatment adherence due to the reduced dosing frequency.

\section{References}

[1] De Geest, S., Borgermans, L., Gemoets, H., Abraham, I., Vlaminck, H., Evers, G., et al. (1995) Incidence, Determinants, and Consequences of Subclinical Noncompliance with Immunosuppressive Therapy in Renal Transplant Recipients. Transplantation, 59, 340-347. http://dx.doi.org/10.1097/00007890-199502000-00006

[2] Butler, J.A., Roderick, P., Mullee, M., Mason, J.C. and Peveler, R.C. (2004) Frequency and Impact of Nonadherence to Immunosuppressants after Renal Transplantation: A Systematic Review. Transplantation, 77, 769-776. http://dx.doi.org/10.1097/01.TP.0000110408.83054.88

[3] Ichimaru, N., Kakuta, Y., Abe, T., Okumi, M., Imamura, R., Isaka, Y., et al. (2008) Treatment Adherence in Renal Transplant Recipients: A Questionnaire Survey on Immunosuppressants. Transplantation Proceedings, 40, $1362-1365$. http://dx.doi.org/10.1016/j.transproceed.2008.02.083

[4] Obi, Y., Ichimaru, N., Kato, T., Kaimori, J.Y., Okumi, M., Yazawa, K., et al. (2013) A Single Daily Dose Enhances the Adherence to Immunosuppressive Treatment in Kidney Transplant Recipients: A Cross-Sectional Study. Clinical and Experimental Nephrology, 17, 310-315. http://dx.doi.org/10.1007/s10157-012-0713-4

[5] Bullingham, R., Monroe, S., Nicholls, A. and Hale, M. (1996) Pharmacokinetics and Bioavailability of Mycophenolate Mofetil in Healthy Subjects after Single-Dose Oral and Intravenous Administration. The Journal of Clinical Pharmacology, 36, 315-324. http://dx.doi.org/10.1002/j.1552-4604.1996.tb04207.x 
Scientific Research Publishing (SCIRP) is one of the largest Open Access journal publishers. It is currently publishing more than 200 open access, online, peer-reviewed journals covering a wide range of academic disciplines. SCIRP serves the worldwide academic communities and contributes to the progress and application of science with its publication.

Other selected journals from SCIRP are listed as below. Submit your manuscript to us via either submit@scirp.org or Online Submission Portal.
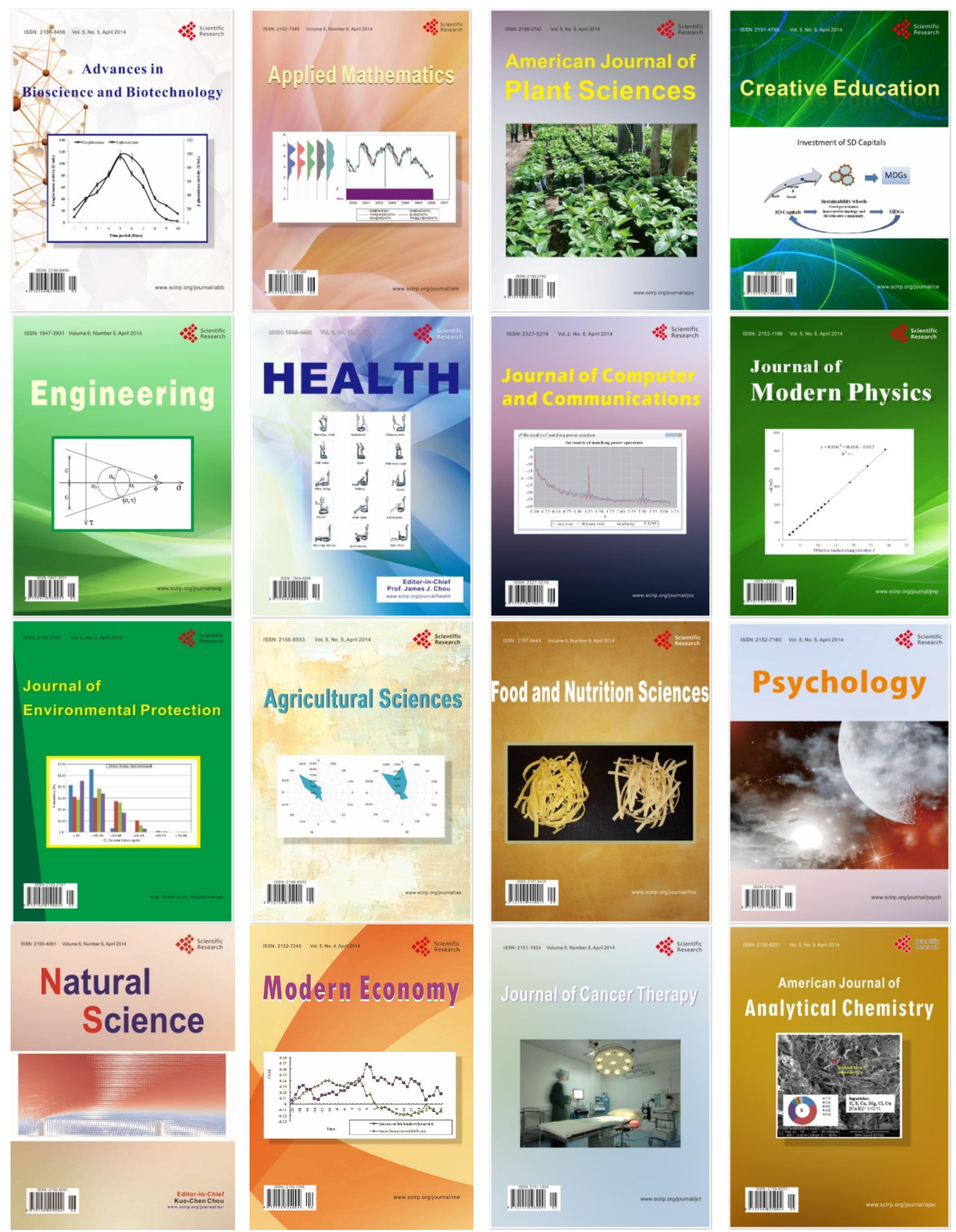